\title{
Clues to cirrhosis - a focus on fibrosis
}

there are
few curative
options
available and
no effective
antifibrotic
therapies

Liver cirrhosis is a major health-care issue worldwide, but there are few curative options available and no effective antifibrotic therapies. Two new research studies focus on fibrosis, a critical step in progressive liver disease leading to cirrhosis, dissecting the cellular and molecular basis of fibrosis at the single-cell level and trialling autologous macrophage therapy in adults with cirrhosis.

In the first study, single-cell RNA sequencing was used to create a transcriptomic profile of over 100,000 human single cells from healthy $(n=5)$ and cirrhotic liver tissue $(n=5)$. The focus was on yielding molecular and cellular definitions for nonparenchymal cell types in the fibrotic niche. "The main driver for initiating this study was my desire to study the fibrotic disease process directly in human liver tissue at high resolution, combined with the serendipitous dawn of single-cell transcriptomics as a tractable technology," explains author Neil Henderson.

Crucially, the researchers identified novel pathogenic and profibrogenic subpopulations of hepatic macrophages (scar-associated $\mathrm{TREM}^{+} \mathrm{CD} 9^{+}$), endothelial cells (ACKR $1^{+}$and PLVAP $^{+}$) and mesenchymal cells (PDGFRA ${ }^{+}$collagen-producing myofibroblasts) in the fibrotic niche of human liver cirrhosis. Moreover,

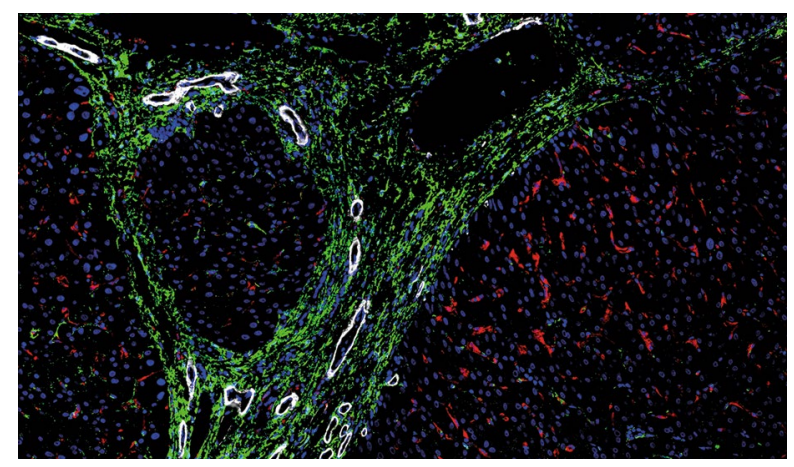

Scar-forming PDGFRA ${ }^{+}$cells (green) within the human liver fibrotic niche. Image courtesy of N. Henderson, University of Edinburgh, UK. the investigators used multi-lineage ligand-receptor modelling to analyse and dissect profibrotic interactions between multiple scar-associated cell lineages and identified several fibrosis-associated molecular pathways (TNFRSF12A, PDGFRA and Notch signalling) that are potentially druggable.

In the second study, a first-inhuman phase I trial, the safety and feasibility of autologous macrophage therapy for liver cirrhosis was examined in nine adults with cirrhosis and Model for End-Stage Liver Disease (MELD) scores of 10-16. Macrophages are crucial cells that take part in the wound healing response, and previous work in mouse models of liver fibrosis had demonstrated that injected macrophages could be beneficial in the liver's response to damage, helping reduce scarring and inflammation and improving regeneration and function. "We have spent time taking these basic findings through to the clinic," clarifies author Stuart Forbes. "We found that blood monocytes (the precursor cells for macrophages) from patients with cirrhosis were different to healthy volunteers, but what was encouraging was finding that these cells could be turned into macrophages that were similar to those from healthy volunteers."

A week before the planned treatment, all participants underwent a standard leukapheresis to collect circulating monocytes, which were then isolated and differentiated into the investigational medical product under good manufacturing practice conditions. Groups of three participants received a single peripheral infusion of autologous macrophages of $10^{7}, 10^{8}$ or up to $10^{9}$ cells. All participants were then assessed for safety outcomes (such as transfusion reactions) and secondary outcomes, which included assessments of liver function (such as MELD score) and noninvasive markers of fibrosis.

The primary outcomes of safety and feasibility were met. Leukapheresis and macrophage infusion were well tolerated and, after 1 year, all participants were still alive and transplant-free, with only one clinical event recorded (minimal ascites). The study was not designed or powered to demonstrate efficacy, but 6 of 9 participants had a reduction in MELD score at 90 days and some improvements in liver fibrosis markers were observed. A phase II randomized controlled trial is now in progress.

The new research adds to our understanding of fibrosis in the context of liver cirrhosis and novel approaches to manage this condition, but more work is needed. "The field of single-cell genomics is incredibly fast moving," notes Henderson, adding that an array of new technologies has emerged, which should help drive forward research and translation to the clinic. "In an era of precision medicine, we hope that these types of approaches will be helpful in informing the design of highly targeted combination therapies that will very likely be necessary to achieve effective antifibrotic potency."

Forbes is also hopeful. "If this macrophage therapy study showed benefit in the treatment arm we would like to develop this as a therapy for liver cirrhosis, which is a major health-care issue with few treatments," he concedes. "We would also like to develop macrophages as a cell therapy in other conditions where there is significant organ injury and little therapeutic options."

Katrina Ray

ORIGINAL ARTICLES Ramachandran, P. et al. Resolving the fibrotic niche of human liver cirrhosis at single-cell level. Nature https:// doi.org/10.1038/s41586-019-1631-3 (2019)| Moroni, F. et al. Safety profile of autologous macrophage therapy for liver cirrhosis. Nat. Med. 25, 1560-1565 (2019) 